\title{
Acquiring Wearable Photoplethysmography Data in Daily Life: The PPG Diary Pilot Study ${ }^{\dagger}$
}

\author{
Peter H. Charlton ${ }^{1,2,3, *}$, Panicos Kyriacou ${ }^{2}$, Jonathan Mant ${ }^{1}$ and Jordi Alastruey ${ }^{3}$ \\ 1 Primary Care Unit, Department of Public Health and Primary Care, University of Cambridge, Cambridge, \\ UK \\ 2 Research Centre for Biomedical Engineering, City, University of London, London, UK \\ 3 Department of Biomedical Engineering, School of Biomedical Engineering and Imaging Sciences, King's \\ College London, King's Health Partners, London, UK \\ * Correspondence: pc657@medschl.cam.ac.uk \\ + Presented at the 7th International Electronic Conference on Sensors and Applications, 15-30 November \\ 2020; Available online: https://ecsa-7.sciforum.net/.
}

Published: 15 November 2020

\begin{abstract}
The photoplethysmogram (PPG) signal is widely measured by smart watches and fitness bands for heart rate monitoring. New applications of the PPG are also emerging, such as to detect irregular heart rhythms, track infectious diseases, and monitor blood pressure. Consequently, datasets of PPG signals acquired in daily life are valuable for algorithm development. The aim of this pilot study was to assess the feasibility of acquiring PPG data in daily life. A single subject was asked to wear a wrist-worn PPG sensor six days a week for four weeks, and to keep a diary of daily activities. The sensor was worn for $75.0 \%$ of the time, signals were acquired for $60.6 \%$ of the time, and signal quality was high for $30.5 \%$ of the time. This small pilot study demonstrated the feasibility of acquiring PPG data during daily living. Key lessons were learnt for future studies: (i) devices which are waterproof and require charging less frequently may provide signals for a greater proportion of the time; (ii) data should either be stored on the device or streamed via a reliable connection to a second device for storage; (iii) it may be beneficial to acquire signals during the night or during periods of low activity to achieve high signal quality; and (iv) there are several promising areas for PPG algorithm development including the design of pulse wave analysis techniques to track changes in cardiovascular properties in daily life. The dataset and code are publicly available at: https://doi.org/10.5281/zenodo.3268500.
\end{abstract}

Keywords: photoplethysmogram; cardiovascular; wearables sensors; pulse wave; daily living

\section{Introduction}

The photoplethysmogram (PPG) signal is widely measured by smart watches and fitness bands. Whilst the PPG is primarily used for heart rate monitoring, the signal contains a wealth of additional information on the cardiovascular, respiratory and autonomic nervous systems. Consequently, the PPG may also be useful for a range of additional applications, such as detecting irregular heart rhythms [1], tracking infectious diseases [2], and monitoring blood pressure [3]. Research into signal processing techniques to analyse the PPG would benefit greatly from publicly available datasets of PPG signals acquired in daily life.

There are several challenges to acquiring PPG data in daily living. Firstly, it is difficult to record PPG signals acquired by a wearable. They must either be stored on a wearable device, or transferred to a second recording device. However, the memory capacity of wearables is often insufficient for prolonged PPG recordings, and Bluetooth connections can be unreliable. Secondly, a wearable needs to be sufficiently comfortable for prolonged use. Thirdly, it needs to be robust enough to the rigors 
of daily life, including being waterproof. Fourthly, the device needs to either have a long battery life, or be charged regularly and reliably. Finally, the wearable must provide the PPG signal rather than solely the derived numerics such as heart rate provided by many commercially available devices. Even wearables designed for use in research do not necessarily meet these requirements [6].

It is important to develop approaches to acquire PPG data in daily life as publicly available PPG datasets are of great value. They provide several benefits including: (i) acting as benchmark data with which to compare techniques developed by different researchers; (ii) facilitating exploratory, hypothesis-generating analyses which do not warrant novel data collection; (iii) making the field accessible to those who cannot acquire their own data; and (iv) aiding reproducibility. Indeed, in a recent review over a third of studies of PPG signal processing techniques used publicly available datasets [4]. Despite these benefits, there are very few publicly available PPG datasets acquired in daily life (the PPG-DaLiA dataset is a notable exception, acquired in close to real-life conditions [5]).

The aim of this study was to assess the feasibility of acquiring PPG data in daily life. A single participant was asked to wear a wearable device for four weeks, and keep a diary of their activities of daily living. The results of the study provide valuable insight into how best to acquire PPG data in daily life to aid future research. In addition, the dataset and analysis code are publicly available.

\section{Methods}

\subsection{Study Participant}

An adult volunteer participated in the study. The participant provided written informed consent to participate. The study was conducted in accordance with the Declaration of Helsinki, and was registered with the King's College London Research Ethics Office (MRA-18/19-10495).

\subsection{Data Collection}

The volunteer was asked to wear a wrist-worn device to acquire PPG signals six days a week, for four weeks. The participant was also asked to record the times and nature of any daily activities, particularly those which may influence the PPG signal (e.g., typewriting, exercise, and sleeping). They were asked to wear the device for the whole day apart from: (i) when recharging the device (approx. 2-3 h per day); (ii) for one day each week for a break; (iii) during activities which could damage the device (such as showering or vigorous exercise); (iv) during any unhygienic activities (such as using the toilet); and (v) if they experienced discomfort or wanted a break.

The SmartCare wrist-worn pulse oximeter (SmartCare Analytics Ltd.) was used as shown in Figure 1. The device acquired red and infrared PPG signals at $100 \mathrm{~Hz}$ at the thumb. The data were streamed to a mobile phone via Bluetooth for storage. The participant was asked to re-connect the device and phone if the Bluetooth disconnected, and record the time of re-connection if possible.

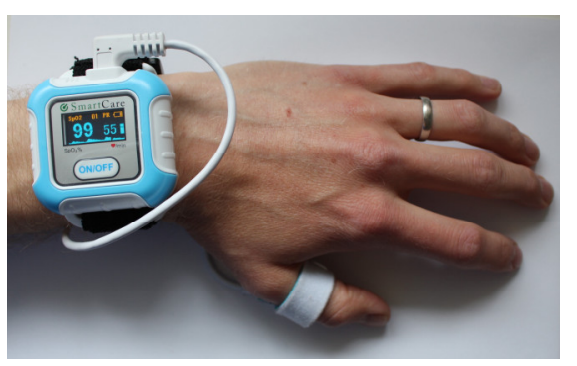

(a)

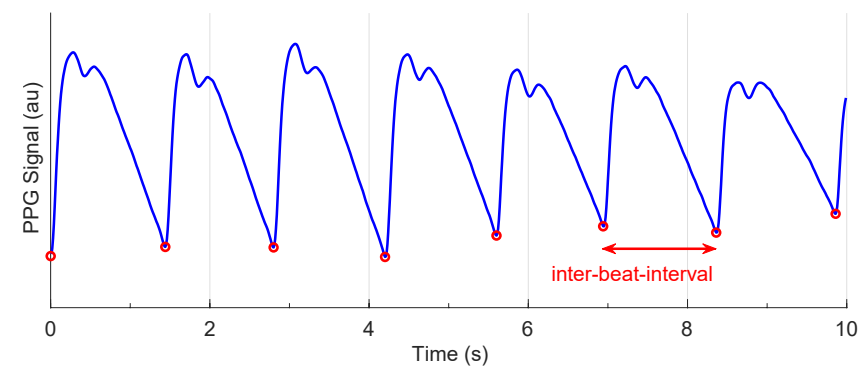

(b)

Figure 1. Acquiring photoplethysmography (PPG) signals in daily living: (a) The SmartCare wristworn pulse oximeter used to record the PPG at the thumb; (b) A sample PPG signal (in arbitrary units), with red circles indicating individual heart beats. 


\subsection{Data Curation and Analysis}

The data were manually reviewed to identify connection and disconnection times which were not noted by the participant, and to correct any misaligned times to within $5 \mathrm{~s}$ of the event.

The infrared PPG signal was used for analysis. The PPG was band-pass filtered with $-3 \mathrm{~dB}$ cutoffs of $0.4 \mathrm{~Hz}$ and $16.8 \mathrm{~Hz}$. Signal quality was assessed using an adaptation of the algorithm described in [7] and coded in PulseAnalyse (v.1.2beta) [8,9]. The heart rates shown in Figure 2 were provided by the device. The heart rates shown in Figure 4 were obtained by: identifying individual heart beats using the algorithm in [10]; calculating beat-to-beat heart rates; and median-filtering the heart rates.

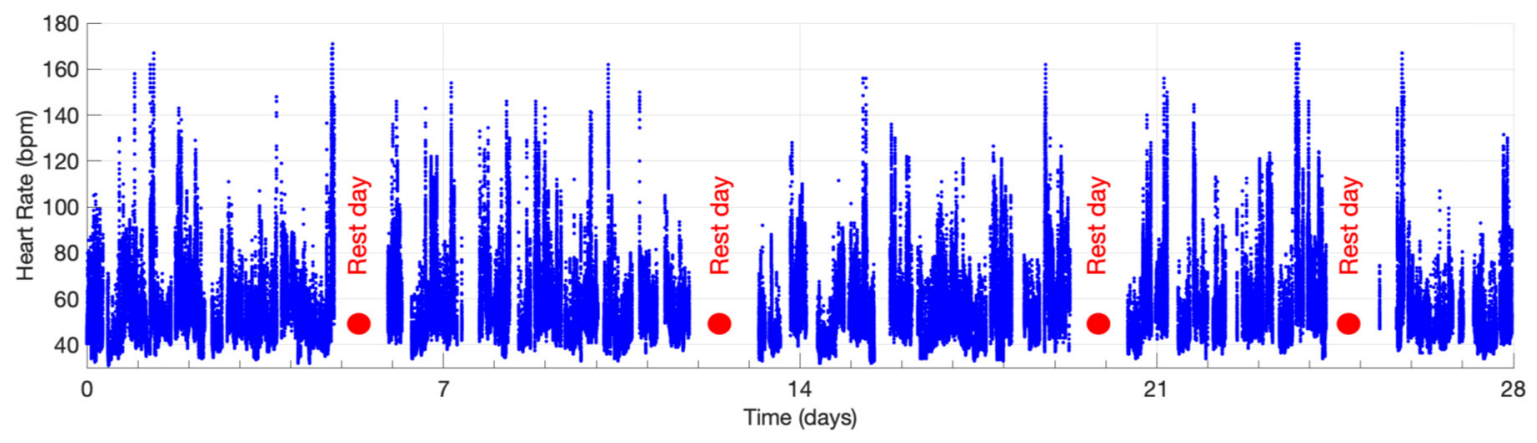

Figure 2. Data were collected from a single volunteer over a period of 28 days including four rest days. Data shown here are heart rates.

\section{Results and Discussion}

The young volunteer (a male in the 18-39 age range) participated for the full 28 days, and PPG data were collected throughout as shown in Figure 2. The study endpoints used to assess the feasibility of acquiring PPG data during daily living are provided in Table 1 . The reasons for data loss and the data quality during different activities of daily living are shown in Figure 3.

\subsection{Device Usage}

The device was worn for $75.0 \%$ of the total possible wear time (18.0 out of 24.0 days), as reported in Table 1. It was primarily removed for charging (13.4\% of the time), as well as for activities involving water, for hygiene purposes, when having a break, and when the participant forgot to reattach the device (see Figure 3a). In this study the sensor probe was located on the thumb and was not waterproof, so it often had to be temporarily removed. In the future it may be beneficial to use a waterproof device which measures the PPG at the wrist to avoid the need to remove it during daily activities such as washing and using the toilet. These changes may also reduce the number of occasions on which participants forget to reattach the device after removing it.

\subsection{Data Capture}

A PPG signal was acquired for $60.6 \%$ of the total possible wear time (349.3 h, i.e., 14.6 days), as detailed in Table 1. PPG data were primarily lost due to dropped Bluetooth connections (accounting for $10.7 \%$ of the time, see Figure 3a). In total 241 Bluetooth disconnections (10.0 per day) were recorded, each requiring the user to manually reconnect the device. This demonstrates the need for reliable wireless connectivity if data are to be streamed from the wearable to a data logging device, and highlights the potential benefit of storing the data on the wearable either permanently or, in a buffer to mitigate against temporary disconnections, to avoid data loss due to connectivity issues.

\subsection{Signal Quality}

Signal quality was high for $30.5 \%$ of the time (Table 1 ) and $50.9 \%$ of the time for which a signal was recorded (Figure 3b). The proportion of the time for which high quality signals were acquired varied greatly between activities as shown in Figure 3b. It was highest during sleep (93.1\% of the time), followed by sedentary activities such as watching TV (55.2\%). It was particularly low during 
exercise (7.4\% during walking and 7.0\% during running) or activities involving hand movement such as preparing food $(9.9 \%)$, cooking $(9.3 \%)$ and housework $(7.6 \%)$. This indicates the potential benefit of acquiring PPG signals during sleep when movement is minimal and the ambient light level is low. It also indicates that PPG signal quality appears to be related to movement, and therefore the signal should preferably be acquired at rest. Indeed, some devices acquire a PPG signal during low activity levels to increase signal quality [11]. Finally, this shows the importance of algorithms to extract parameters from the PPG accurately even in the presence of motion artifact [12].

Table 1. The data capture and data quality results, shown as the time and percentage of the maximum possible time.

\section{Endpoint}

Time, Hours (\%)

\begin{tabular}{lc}
\hline Total possible wear time: the time for which the participant was asked to wear the device & $576.8(100.0)$ \\
Wear time: the time for which the participant noted they wore the device & $432.3(75.0)$ \\
Signal time: the time for which a PPG signal was acquired & $349.3(60.6)$ \\
High quality signal time: the time for which a high quality signal was acquired & $176.1(30.5)$ \\
\hline
\end{tabular}

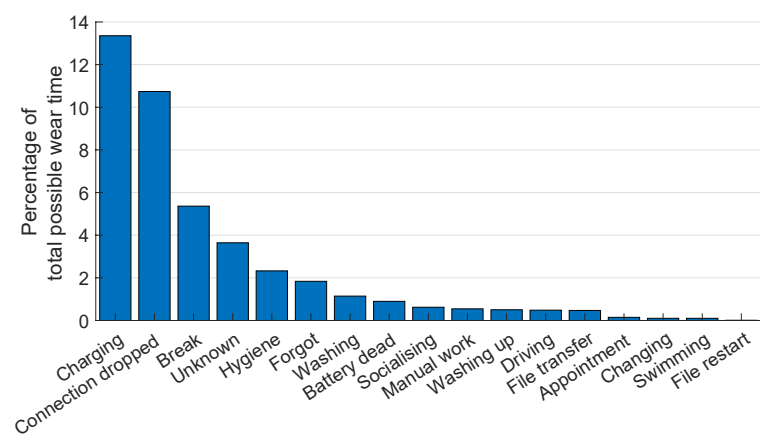

(a)

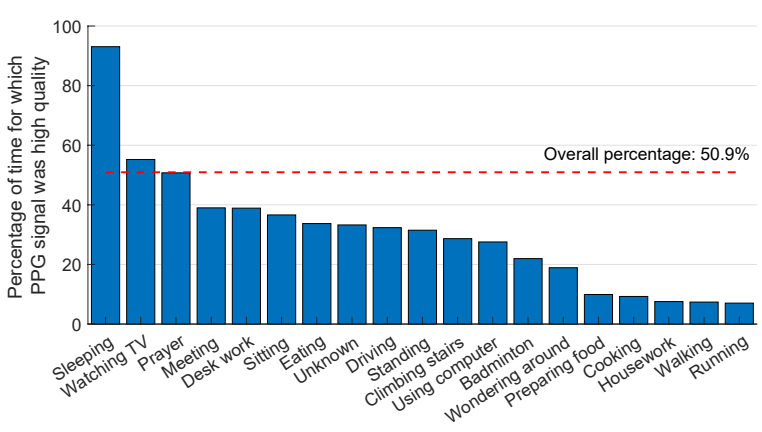

(b)

Figure 3. Data capture and data quality: (a) Reasons for data loss; (b) The data quality associated with different activities of daily living.

\subsection{Potential Utility for Pulse Wave Analysis}

The PPG signals acquired by the device often exhibited the distinct systolic and diastolic peaks on each pulse wave which are associated with young, healthy subjects (as shown in Figure 1b). This indicates that the device may be suitable for pulse wave analysis studies. Pulse wave shape changed considerably during activities of daily living. For instance, Figure 4a shows the changes as heart rate reduced during recovery from exercise, with the amplitude of the diastolic peak increasing over time. Figure $4 \mathrm{~b}$ shows the changes in the first $30 \mathrm{~min}$ of sleep, with the amplitude of the systolic peak decreasing over time. These may indicate that cardiovascular properties such as arterial stiffness or blood pressure changed during the activities. The examples illustrate potential areas for exploratory analyses to develop techniques to assess cardiovascular state from the PPG in daily life.

\subsection{Limitations and Future Work}

The key limitations to this study are that data were acquired from a single participant, using a single device. Therefore, the findings may not be generalisable. Different people may conduct different activities during daily life, and in different ways. In addition, devices for acquiring PPG signals are being developed continuously, and new devices may provide even better performance than the current device. Activities of daily living were self-reported in this study: future studies may consider the use of body-worn cameras to obtain gold standard labels of activities. 


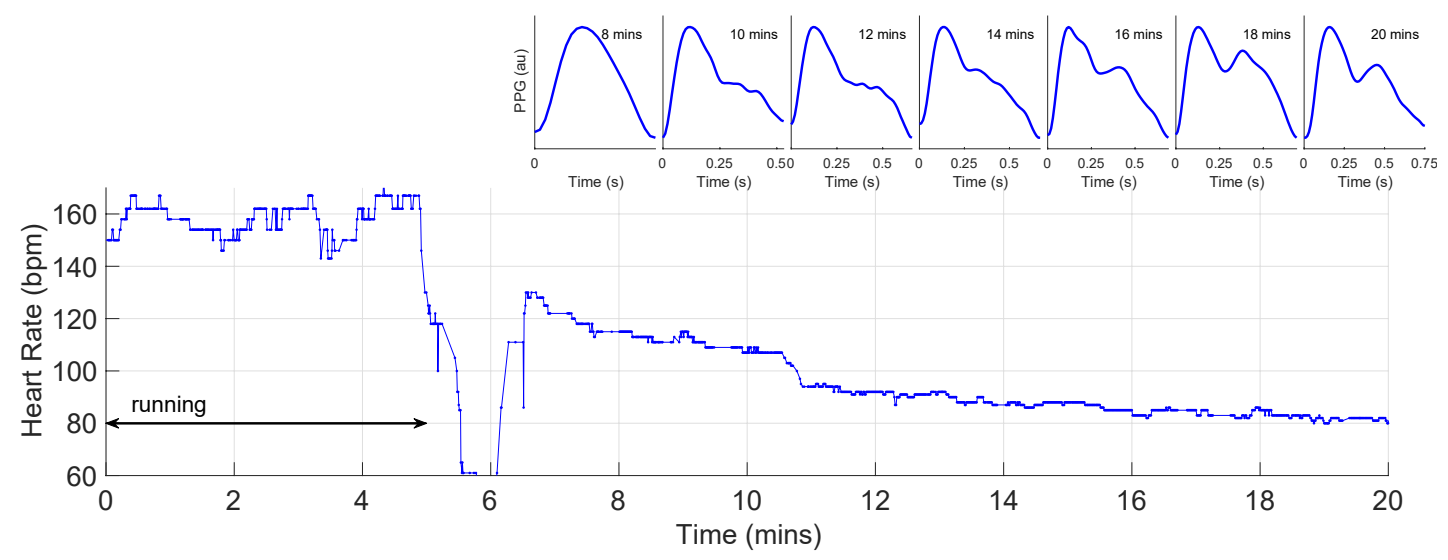

(a)

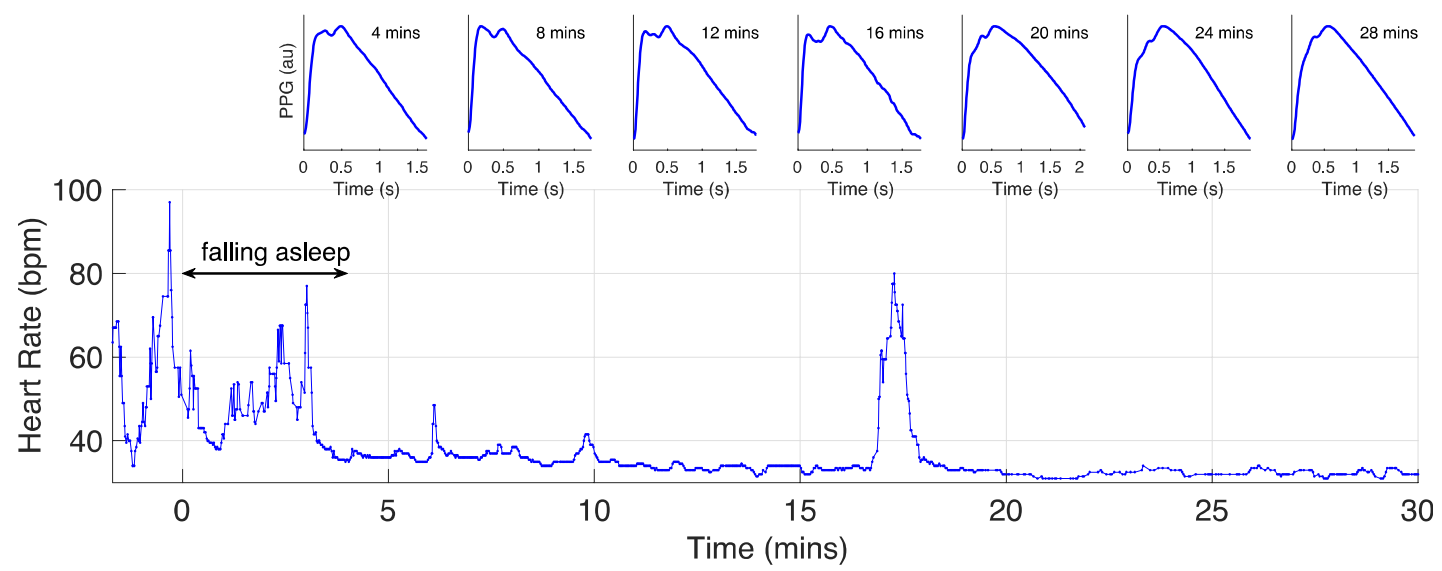

(b)

Figure 4. Changes in PPG pulse wave shape during activities of daily living: (a) during recovery from exercise; (b) whilst falling sleep.

This study provides several directions for future research. Future research should assess whether the findings of this study are consistent between participants and between devices. In particular, it is important to assess whether PPG signal quality is closely related to the level of movement. If so, then devices could be designed to only record PPG signals during low movement levels, thus extending battery life and reducing memory consumption.

\section{Conclusions}

This small pilot study demonstrated the feasibility of acquiring PPG data during daily living. Key lessons were learnt for future studies: (i) devices which are waterproof and require charging less frequently may acquire signals for a greater proportion of the time; (ii) data should either be stored on the device or streamed via a reliable connection to a second device for storage; (iii) it may be beneficial to acquire signals during the night or during periods of low activity to achieve high signal quality; and (iv) there are several promising areas for PPG algorithm development including the design of pulse wave analysis techniques to track changes in cardiovascular properties in daily life. The dataset and analysis code are publicly available at: https://doi.org/10.5281/zenodo.3268500; and further details are provided at: https://peterhcharlton.github.io/ppg-diary.

Funding: This work was supported by British Heart Foundation (BHF) grants [PG/15/104/31913] and [FS/20/20/34626], the Wellcome EPSRC Centre for Medical Engineering at King's College London [WT 203148/Z/16/Z], and a BioMedEng18 conference award to PHC. The authors acknowledge: financial support from the Department of Health through the National Institute for Health Research (NIHR) Cardiovascular MedTech Co-operative at Guy's and St Thomas' NHS Foundation Trust. The views expressed are those of the author(s) and not necessarily those of the BHF, Wellcome Trust, EPSRC or NIHR. 
Conflicts of Interest: The authors declare no conflict of interest. The funders had no role in the design of the study; in the collection, analyses, or interpretation of data; in the writing of the manuscript, or in the decision to publish the results.

\section{References}

1. Pereira, T.; Tran, N.; Gadhoumi, K.; Pelter, M.M.; Do, D.H.; Lee, R.J.; Colorado, R.; Meisel, K.; Hu, X. Photoplethysmography based atrial fibrillation detection: A review. NPJ Digit. Med. 2020, 3, 3, doi:10.1038/s41746-019-0207-9.

2. Radin, J.M.; Wineinger, N.E.; Topol, E.J.; Steinhubl, S.R. Harnessing wearable device data to improve statelevel real-time surveillance of influenza-like illness in the USA: A population-based study. Lancet Digit. Health 2020, 2, e85-e93, doi:10.1016/S2589-7500(19)30222-5.

3. Elgendi, M.; Fletcher, R.; Liang, Y.; Howard, N.; Lovell, N.H.; Abbott, D.; Lim, K.; Ward, R. The use of photoplethysmography for assessing hypertension. NPJ Digit. Med. 2019, 2, 60, doi:10.1038/s41746-0190136-7.

4. Charlton, P.H.; Birrenkott, D.A.; Bonnici, T.; Pimentel, M.A.F.; Johnson, A.E.W.; Alastruey, J.; Tarassenko, L.; Watkinson, P.J.; Beale, R.; Clifton, D.A. Breathing rate estimation from the electrocardiogram and photoplethysmogram: A review. IEEE Rev. Biomed. Eng. 2018, 11, 2-20, doi:10.1109/RBME.2017.2763681.

5. Reiss, A.; Indlekofer, I.; Schmidt, P.; Van Laerhoven, K. Deep PPG: large-scale heart rate estimation with convolutional neural networks. Sensors 2019, 19, 3079, doi:10.3390/s19143079.

6. Bonnici, T.; Orphanidou, C.; Vallance, D.; Darrell, A.; Tarassenko, L. Testing of wearable monitors in a realworld hospital environment: What lessons can be learnt? In Proceedings of the Conf Proc 9th Wearable and Implantable BSNs, London, UK, 9-12 May 2012; pp. 79-84.

7. Orphanidou, C.; Bonnici, T.; Charlton, P.; Clifton, D.; Vallance, D.; Tarassenko, L. Signal-quality indices for the electrocardiogram and photoplethysmogram: Derivation and applications to wireless monitoring. IEEE J. Biomed. Health Inform. 2015, 19, 832-838, doi:10.1109/JBHI.2014.2338351.

8. Charlton, P.H.; Mariscal Harana, J.; Vennin, S.; Li, Y.; Chowienczyk, P.; Alastruey, J. Modeling arterial pulse waves in healthy aging: A database for in silico evaluation of hemodynamics and pulse wave indexes. Am. J. Physiol. Circ. Physiol. 2019, 317, H1062-H1085, doi:10.1152/ajpheart.00218.2019.

9. Charlton, P.H. PulseAnalyse: A Signal Processing Tool for Cardiovascular Pulse Waves. Available online: https://zenodo.org/record/3272123/export/dcat\#.X6OLF1ARXIU (accessed on 19 October 2020).

10. Aboy, M.; McNames, J.; Thong, T.; Tsunami, D.; Ellenby, M.S.; Goldstein, B. An automatic beat detection algorithm for pressure signals. IEEE Trans. Biomed. Eng. 2005, 52, 1662-1670, doi:10.1109/TBME.2005.855725.

11. Perez, M. V.; Mahaffey, K.W.; Hedlin, H.; Rumsfeld, J.S.; Garcia, A.; Ferris, T.; Balasubramanian, V.; Russo, A.M.; Rajmane, A.; Cheung, L.; et al. Large-scale assessment of a smartwatch to identify atrial fibrillation. N. Engl. J. Med. 2019, 381, 1909-1917, doi:10.1056/NEJMoa1901183.

12. Zhang, Z.; Pi, Z.; Liu, B. TROIKA: A general framework for heart rate monitoring using wrist-type photoplethysmographic signals during intensive physical exercise. IEEE Trans. Biomed. Eng. 2015, 62, 522531, doi:10.1109/TBME.2014.2359372.

Publisher's Note: MDPI stays neutral with regard to jurisdictional claims in published maps and institutional affiliations.

C 2020 by the authors. Submitted for possible open access publication under the terms and conditions of the Creative Commons Attribution (CC BY) license (http://creativecommons.org/licenses/by/4.0/). 